\title{
Klinische Immunintervention: aktuelle und künftige Ansätze
}

Bibliografie

DOI http://dx.doi.org/ 10.1055/s-0032-1324896

Arzneimittelforschung 2012;

62, Suppl. 1: S1-S2 @ Georg

Thieme Verlag KG Stuttgart .

New York · ISSN 0004-4172

Korrespondenzadresse

Prof. Dr. med. Stefan Endres

Ludwig-Maximilians-Universität

München

Ziemssenstraße 1

80336 München

endres@Imu.de
Drei große Bereiche der Medizin erschließen sich der klinischen Immunintervention: die Tumorerkrankungen, die entzündlichen Erkrankungen inklusive der Autoimmunkrankheiten und die Transplantationsmedizin. Für das Paul-MartiniSymposium 2012 konnten herausragende Mediziner und Wissenschaftler in jedem dieser Bereiche gewonnen werden, die eine Bestandsaufnahme über neue immunstimulierende wie suppressive Therapien aus diesen 3 Fachgebieten vornehmen.

„Cancer immunotherapy comes of age“ - so der Titel zweier Übersichtsarbeiten, die vor Kurzem fast zeitgleich in den Zeitschriften Nature und Journal of Clinical Oncology erschienen. In der Tat ist es seit Langem ein Ziel, das körpereigene Immunsystem gegen Tumoren zu richten. Mit der erstmaligen Zulassung eines therapeutischen Antikörpers, der gegen Tumorzellen gerichtete TZellen „entfesselt“, ist 2011 ein wichtiger Meilenstein auf diesem Weg erreicht worden. Das rasant gewachsene Verständnis von Immunität, immunologischer Toleranz und Immunsuppression beginnt, therapeutische Früchte zu tragen.

In den späten 1990er-Jahren begann der Einzug der tumorspezifischen monoklonalen Antikörper in die Krebstherapie. Heute sind hierfür in Deutschland 9 verschiedene Antikörper zugelassen: zur Therapie von soliden Tumoren (Mamma-, Kolorektal-, Ovarial- und Magenkarzinom, Melanomen und Kopf-Hals-Tumore), von NonHodgkin-Lymphomen und von Leukämien.

Das Symposium wird eröffnet mit 2 vielversprechenden Weiterentwicklungen tumorgerichteter monoklonaler Antikörper: erstens den bispezifischen Antikörpern, die die Immuneffektorzelle in unmittelbaren physischen Kontakt mit der Tumorzelle bringen und sie dort aktivieren; und zweitens der gezielten Glykosierung monoklonaler Antikörper zur Optimierung ihrer Pharmakokinetik und Wirksamkeit. Komplementär zur „passiven“ Immuntherapie mit Antikörpern ist die aktive Vakzinierung mit Tumorantigenen: Zur peptidbasierten Tumor-Vakzinierung erschien 2012 in Nature Medicine eine viel beachtete Publikation über zwei aussichtsreiche Studien mit Patienten mit Nierenzellkarzinom. Besonders erfreulich: Bei der Entwicklung dieser beiden ImmuntherapieStrategien - bispezifische Antikörper und TumorPeptid-Vakzinierung - sind Gruppen aus Deutschland unter Zusammenarbeit von akademischer Forschung und Industrie international führend. Eine zentrale Effektorzelle in der Tumorimmuntherapie ist die zytotoxische T-Zelle. Hier setzen 2 Therapiestrategien direkt an: die adoptive T-
Zelltherapie, bei der körpereigene tumorspezifische T-Zellen ex vivo expandiert, modifiziert und dann dem Patienten re-infundiert werden, und der Einsatz immunmodulatorischer Antikörper wie des Anti-CTLA-4-Antikörpers, der das inhibitorische Oberflächenprotein „cytotoxic T lymphocyte antigen-4“ auf T-Zellen blockiert. Damit wird, bildlich gesprochen, „die Handbremse“ auf diesen zentralen Effektorzellen gelöst. Ein erster solcher Antikörper ist seit 2011 für die Therapie des metastasierten malignen Melanoms zugelassen.

Interleukin-1, in den 1980er-Jahren als erstes einer heute über 30 Proteine umfassenden Zytokinfamilie entdeckt, ist ein prototypisches Target für die zielgerichtete Therapie entzündlicher Erkrankungen. Eine rekombinant hergestellte Variante des natürlichen Interleukin-1-Rezeptor-Antagonisten ist für die Therapie der rheumatoiden Arthritis zugelassen. Weitere Indikationen am Horizont für Interleukin-1-antagonistische Therapie umfassen ein erstaunlich breites Spektrum von seltenen periodischen Fiebersyndromen bis hin zu Diabetes mellitus und Arteriosklerose.

Die Entdeckung der Toll-like-Rezeptoren des angeborenen Immunsystems wurde im vergangenen Jahr mit dem Nobelpreis für Medizin ausgezeichnet. Die Toll-like-Rezeptoren und weitere intrazelluläre Rezeptorfamilien erkennen bakterielle und virale Nukleinsäuren und andere mikrobielle Bausteine. Bei Infektionen lösen sie die erwünschte antimikrobielle Immun- und Entzündungsantwort aus. Das lässt sich nutzen für die Beschleunigung und Steigerung der Wirkung von Aktivimpfstoffen. Umgekehrt bilden diese Rezeptoren aber auch neue, attraktive Targets für die therapeutische Eindämmung von Entzündungen bei chronischen entzündlichen und Autoimmunerkrankungen.

Schließlich die Organtransplantation: Die Immunsuppression zur Verhinderung der Transplantatabstoßung war die erste Indikation überhaupt für die therapeutische Gabe von monoklonalen Antikörpern in der Medizin. Auch in den neuesten Immuntherapie-Regimen zeigt sich mit besonderer Prägnanz ein wichtiges Prinzip: dass oft die Kombination verschiedener Ansätze, etwa von klassischem Immunsuppressivum wie Azathioprin und zielgerichtetem therapeutischem Antikörper, die besten Ergebnisse erzielt.

Ein besonderer Reiz und eine Chance dieses Symposiums ist es, dass hier die neuesten Entwicklungen der Immuntherapie auf allen 3 Feldern Tumoren, Entzündung und Transplantation - gemeinsam und nebeneinander betrachtet werden. 
Hier wird die erstaunliche „therapeutische Nähe“ dieser sonst getrennten medizinischen Felder deutlich. Dies zeigt sich auch in der jüngst von einem Unternehmen getroffen, umstrittenen Entscheidung, einen für die Therapie einer Leukämieform seit mehreren Jahren zugelassenen monoklonalen Antikörper vom Markt zu nehmen und ihn zugleich gegen Multiple Sklerose, eine entzündliche Erkrankung, zur Zulassung einzureichen.

Die therapeutischen Antikörper, die Vakzinierungen und insbesondere die zellbasierten Therapien erfordern in besonderem Maße die Zusammenarbeit von Kliniken, Universitäten, forschender Industrie und der regulatorischen Behörden. Auch dafür bietet die Tradition der Paul-Martini-Stiftung, Vertreterinnen und Vertreter aus allen diesen 4 Bereichen zur Diskussion zusammenzubringen, ein fruchtbares Forum. Das Symposium soll somit zu einem offenen Diskurs zwischen universitärer und industrieller Forschung beitragen und damit Impulse für innovative Behandlungskonzepte geben. Es ist deshalb erfreulich, dass neben Wissenschaftlern aus den Universitäten und der Industrie auch Sachverständige aus Ministerien, Behörden und Verbänden zusammenkommen.
Für das Gelingen des Symposiums haben sich viele engagiert: An erster Stelle natürlich die Referenten, aber auch der Vorstand der Paul-Martini-Stiftung und die Geschäftsstelle, die dieses Symposium vorbereitet und organisatorisch auf die Beine gestellt haben. Ebenso danken wir der Deutschen Akademie der Naturforscher Leopoldina, die es mit uns durchführt. Nun hoffen wir, dass das Symposium - nicht zuletzt auch dank der Diskussionsbeiträge dazu beiträgt, dass das Verständnis für die beeindruckenden Möglichkeiten der Immuntherapie vertieft und damit letztlich den Patienten, die an Tumor- und Entzündungserkrankungen leiden oder organtransplantiert sind, noch besser geholfen werden kann.

\section{Prof. Dr. med. Stefan Endres}

Ludwig-Maximilians-Universität München

Prof. Dr. med. Stefan Meuer

Ruprecht-Karls-Universität Heidelberg

Prof. Dr. med. Dr. h.c. Peter C. Scriba

Ludwig-Maximilians-Universität München 